\title{
RAYLEIGH SCATTERING FOR THE KELVIN-INVERTED ELLIPSOID
}

\author{
BY \\ GEORGE DASSIOS (Division of Applied Math., Dept. of Chemical Engineering, University of \\ Patras, Greece) \\ AND
}

TOUVIA MILOH (Dept. of Fluid Mechanics and Heat Transfer, School of Engineering, Tel-Aviv University, Israel)

\begin{abstract}
The Kelvin-inverted ellipsoid, with the center of inversion at the center of the ellipsoid, is a nonconvex biquadratic surface that is the image of a triaxial ellipsoid under the Kelvin mapping. It is the most general nonconvex 3-D body for which the Kelvin inversion method can be used to obtain analytic solutions for low-frequency scattering problems. We consider Rayleigh scattering by such a fourth-degree surface and provide all relevant analytical calculations possible within the theory of ellipsoidal harmonics. It is shown that only ellipsoidal harmonics of even degree are needed to express the capacity of the inverted ellipsoid. Special cases of prolate or oblate spheroids and that of the sphere are recovered through appropriate limiting processes. The crucial calculations of the norm integrals, which are expressible in terms of known ellipsoidal harmonics, are outlined in Appendix B.
\end{abstract}

1. Introduction. Rayleigh initiated the theory of low-frequency scattering and provided the first analytical results for a triaxial ellipsoidal scatterer in 1897 [13]. Rayleigh's results were based on physical and dimensional arguments. The first systematic study of low-frequency scattering was proposed by Stevenson [12] in 1953. Since then a large amount of work has been published and extended references can be found in $[3,6,15]$ and references therein.

Almost all the analytical results available for low-frequency scatterers were obtained for simple convex bodies with the ellipsoid as the most complicated one. This is so because the ellipsoidal coordinate system is the most general quadratic system that renders a separable solution for Laplace's equation. In [3], a technique was proposed, which is based on the property of the Kelvin inversion [14] to preserve harmonicity and through which it was possible to obtain the Rayleigh approximation for scattering by a nonconvex body in the shape of a peanut or a blood cell [4]. The peanut shape is

Received December 30, 1997.

1991 Mathematics Subject Classification. Primary 35A30, 35A35, 35C10, 35J05.

(C)1999 Brown University 
the image of an oblate spheroid under Kelvin inversion, while the shape of a blood cell is the image of a prolate spheroid under Kelvin inversion. The crucial step in the Kelvin inversion method $[3,4]$ is connected to the possibility of using an eigenfunction expansion for the fundamental solution of Laplace's operator. For the case of a triaxial ellipsoid such an expansion was obtained in $[8,9,10,11,16]$. In the present work we combine the method introduced in [3] and the above eigenfunction expansion to solve the low-frequency scattering problem by an inverted triaxial ellipsoid that is a nonconvex body with an equation quadratic in the cartesian variables $x_{1}^{2}, x_{2}^{2}$, and $x_{3}^{2}$. The inverted ellipsoid, which is a fourth-degree surface, generalizes the results for nonconvex scatterers obtained in [4] to a genuine 3-D shape. Since the triaxial ellipsoid is the most general coordinate system in which Laplace's equation is separable, the inverted ellipsoid is the most general nonconvex body for which an analytic scattering solution is possible.

As is well known, $[3,6]$, for the case of a soft scatterer, the leading two far-field approximations can be expressed in terms of the capacity of the body and therefore all we need to evaluate is the capacity of the inverted ellipsoid. Although some estimates for the capacity of such a body are already known [2], no exact form has been given before. Furthermore, all the integrals that appear in the expression for the capacity and which are expressible in terms of ellipsoidal harmonics of degree less than or equal to two, are evaluated in closed form. These are surface norm integrals and their evaluation, which is based on some elegant properties of the ellipsoidal harmonics, forms by no means a straightforward task. It is recalled that no ellipsoidal harmonic of degree greater than or equal to four is known in reasonable form, since the exact values of the constants involved are given as roots of some irreducible polynomials of degree greater than or equal to three $[5,7]$. An appendix that summarizes all completely known ellipsoidal harmonics is included for immediate reference.

2. Low-frequency scattering by an inverted ellipsoid. Let $S$ denote the surface of a triaxial ellipsoid

$$
\frac{x_{1}^{2}}{a_{1}^{2}}+\frac{x_{2}^{2}}{a_{2}^{2}}+\frac{x_{3}^{2}}{a_{3}^{2}}=1,
$$

where we assume that $0<a_{3} \leq a_{2} \leq a_{1}<+\infty$ and $\left(x_{1}, x_{2}, x_{3}\right)$ stand for Cartesian coordinates. The Kelvin transformation

$$
\mathbf{r} \rightarrow \mathbf{r}^{\prime}=\frac{\mathbf{r}}{r^{2}}
$$

with $\mathbf{r}=\left(x_{1}, x_{2}, x_{3}\right), r=\sqrt{x_{1}^{2}+x_{2}^{2}+x_{3}^{2}}$, and $\mathbf{r}^{\prime}=\left(x_{1}^{\prime}, x_{2}^{\prime}, x_{3}^{\prime}\right)$ maps the ellipsoid (1) into the inverted ellipsoid $S^{\prime}$ given by

$$
\frac{x_{1}^{\prime 2}}{a_{1}^{2}}+\frac{x_{2}^{\prime 2}}{a_{2}^{2}}+\frac{x_{3}^{\prime 2}}{a_{3}^{2}}=\left(x_{1}^{\prime 2}+x_{2}^{\prime 2}+x_{3}^{\prime 2}\right)^{2}
$$

which is a nonconvex biquadratic surface. Note that the origin, which is the Kelvin image of a point at infinity, is excluded from $S^{\prime}$ since the form of (3) involves a multiplication of the exact Kelvin image of (1) by the factor $\mathbf{r}^{\prime \prime}$.

The following figures show for example an inverted ellipsoid with $a_{1}=3 . a_{2}=2$, $a_{3}=1$. 


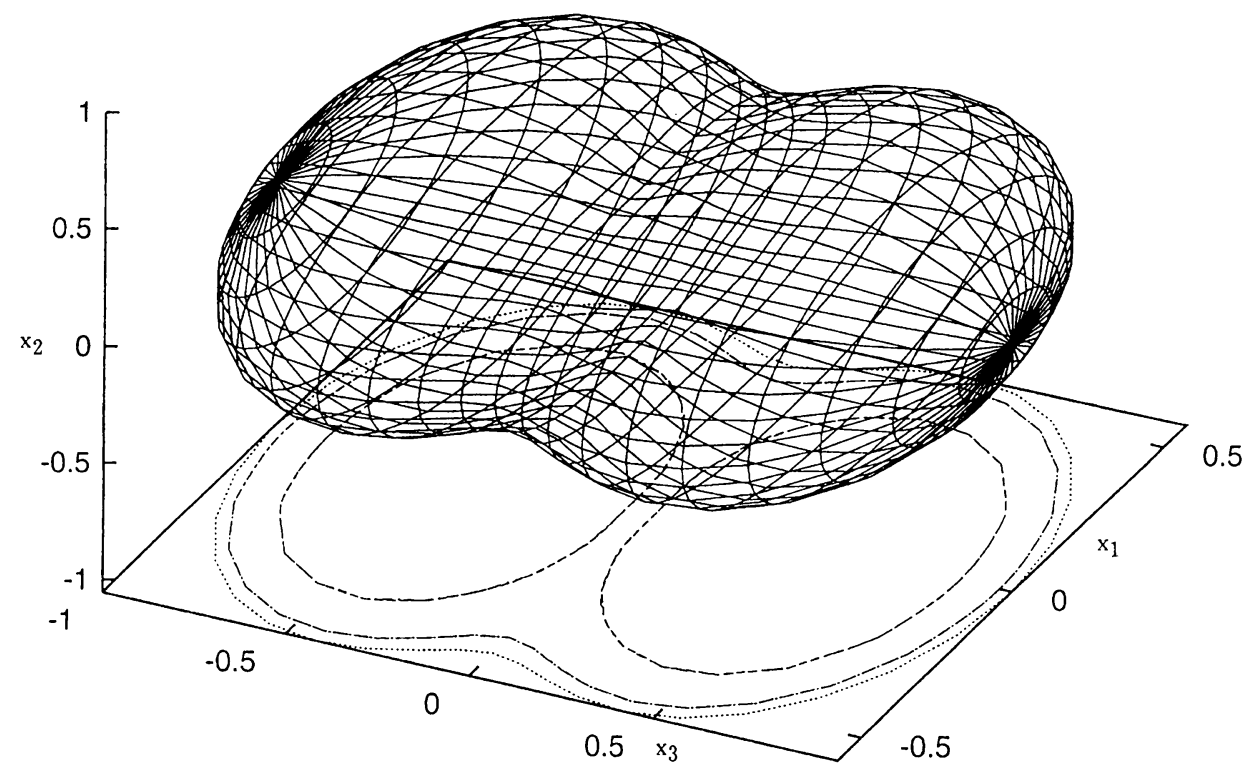

FIG. 1. Complete Kelvin-inverted ellipsoid with $a_{1}=3, a_{2}=2, a_{1}=1$

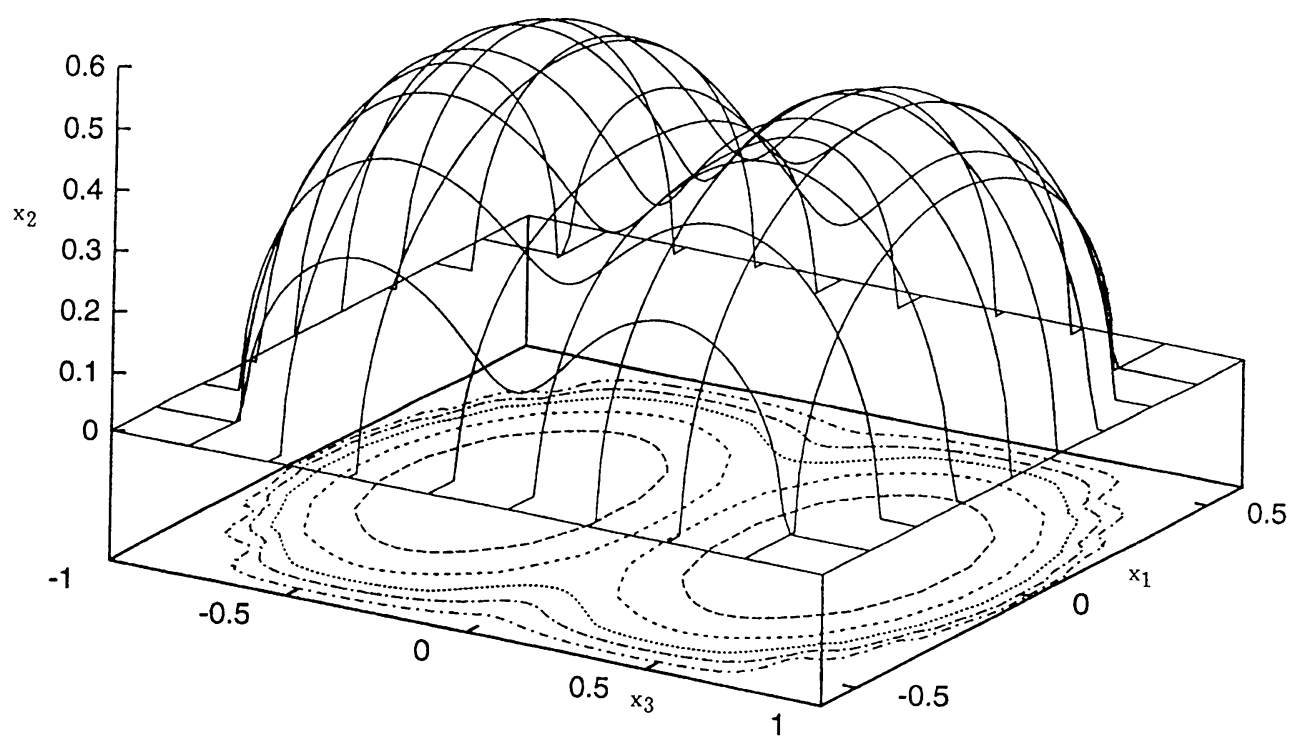

FIG. 2. The half-space $x_{2}>0$ of Fig. 1

Let us now consider the scattering problem of a time harmonic plane wave $\exp \{-i \omega t+$ $\left.i \mathbf{k} \cdot \mathbf{r}^{\prime}\right\}$ by an acoustically soft body in the shape of the inverted ellipsoid $S^{\prime}$. Here $\omega$ denotes the frequency and $k$ is the wave-number of the ambient wave. The scattered field $u^{s}$ is then determined by the solution of the following exterior boundary value problem 
$[3,4]$ :

$$
\begin{gathered}
\left(\Delta^{\prime}+k^{2}\right) u^{s}\left(\mathbf{r}^{\prime}\right)=0, \quad \text { outside } S^{\prime}, \\
u^{s}\left(\mathbf{r}^{\prime}\right)=-e^{i \mathbf{k} \cdot \mathbf{r}^{\prime}}, \quad \text { on } S^{\prime}, \\
\left(\frac{\partial}{\partial r^{\prime}}-i k\right) u^{s}\left(\mathbf{r}^{\prime}\right)=O\left(\frac{1}{r^{\prime 2}}\right), \quad \text { as } r^{\prime} \rightarrow \infty
\end{gathered}
$$

where (6) holds uniformly in all directions.

In the far field we obtain the asymptotic form

$$
u^{s}\left(\mathbf{r}^{\prime}\right)=g\left(\hat{\mathbf{r}^{\prime}}, \hat{\mathbf{k}}\right) h\left(k r^{\prime}\right)+O\left(\frac{1}{r^{\prime 2}}\right)
$$

where

$$
h(x)=h_{0}^{(1)}(x)=\frac{e^{i x}}{i x} .
$$

It provides the fundamental solution of the Helmholtz operator and $g\left(\hat{\mathbf{r}}^{\prime}, \hat{\mathbf{k}}\right)$ denotes the normalized scattering amplitude, i.e., the response of the scatterer in the direction $\hat{\mathbf{r}}^{\prime}$ to a plane wave excitation propagating along $\hat{\mathbf{k}}$. As is well known [3], in the low-frequency approximation the scattering amplitude assumes the form

$$
g\left(\hat{\mathbf{r}^{\prime}}, \hat{\mathbf{k}}\right)=-i k C^{\prime}-k^{2} C^{\prime 2}+O\left(k^{3}\right),
$$

where

$$
C^{\prime}=-\frac{1}{4 \pi} \oint_{S^{\prime}} \frac{\partial u^{\prime}\left(\mathbf{r}^{\prime}\right)}{\partial n^{\prime}} d s\left(\mathbf{r}^{\prime}\right)
$$

denotes the capacity of the surface $S^{\prime}$, and the unit normal vector on $S^{\prime}$ is taken in the outwards direction. The capacity potential $u^{\prime}$ represents the solution of the following exterior boundary value problem:

$$
\begin{aligned}
\Delta u^{\prime}\left(\mathbf{r}^{\prime}\right) & =0, \quad \text { outside } S^{\prime}, \\
u^{\prime}\left(\mathbf{r}^{\prime}\right) & =1, \quad \text { on } S^{\prime}, \\
u^{\prime}\left(\mathbf{r}^{\prime}\right) & =O\left(\frac{1}{r^{\prime}}\right), \quad \text { as } r^{\prime} \rightarrow+\infty .
\end{aligned}
$$

It was shown in [3] that the value of the capacity can also be obtained from the limiting relation

$$
C^{\prime}=\lim _{r \rightarrow 0^{+}} u(\mathbf{r}),
$$

where the inverted capacity potential $u$ solves the following interior boundary value problem:

$$
\begin{gathered}
\Delta u(\mathbf{r})=0, \quad \text { inside } S, \\
u(\mathbf{r})=\frac{1}{r}, \quad \text { on } S,
\end{gathered}
$$

with $S$ being the preimage (1) of $S^{\prime}$ under the Kelvin mapping (2).

In other words, instead of solving the exterior problem defined in (11)-(13) for the complicated surface $S^{\prime}$, it is sufficient to solve the interior problem given by (15)-(16) 
for the much simpler surface $S$. This is the essence of the Kelvin technique applied to low-frequency scattering theory.

In order to solve the boundary value problem postulated in (15)-(16), we introduce the triply orthogonal ellipsoidal coordinates $[5,7](\rho, \mu, \nu)$ that are connected to the Cartesian coordinates $\left(x_{1}, x_{2}, x_{3}\right)$ by

$$
\begin{aligned}
& x_{1}=\frac{h_{1}}{h_{1} h_{2} h_{3}} \rho \mu \nu, \\
& x_{2}=\frac{h_{2}}{h_{1} h_{2} h_{3}} \sqrt{\rho^{2}-h_{3}^{2}} \sqrt{\mu^{2}-h_{3}^{2}} \sqrt{h_{3}^{2}-\nu^{2}}, \\
& x_{3}=\frac{h_{3}}{h_{1} h_{2} h_{3}} \sqrt{\rho^{2}-h_{2}^{2}} \sqrt{h_{2}^{2}-\mu^{2}} \sqrt{h_{2}^{2}-\nu^{2}},
\end{aligned}
$$

where

$$
\begin{aligned}
& h_{1}^{2}=a_{2}^{2}-a_{3}^{2}, \\
& h_{2}^{2}=a_{1}^{2}-a_{3}^{2}, \\
& h_{3}^{2}=a_{1}^{2}-a_{2}^{2}
\end{aligned}
$$

are the squares of the main semifocal distances and

$$
0 \leq \nu^{2} \leq h_{3}^{2} \leq \mu^{2} \leq h_{2}^{2} \leq \rho^{2}<+\infty .
$$

Separation of variables for Laplace's equation in the ellipsoidal coordinate system leads to the complete and orthogonal system of ellipsoidal harmonics which assume the form

$$
\mathbb{E}_{n}^{m}(\rho, \mu, \nu)=E_{n}^{m}(\rho) E_{n}^{m}(\mu) E_{n}^{m}(\nu)
$$

for interior regions $\rho<a_{1}$ and

$$
\mathbb{F}_{n}^{m}(\rho, \mu, \nu)=F_{n}^{m}(\rho) E_{n}^{m}(\mu) E_{n}^{m}(\nu)
$$

for exterior regions $\rho>a_{1}$. The functions $E_{n}^{m}$ and $F_{n}^{m}$, with $n=0,1,2, \ldots$ and $m=$ $1,2, \ldots, 2 n+1$, are the interior and the exterior Lamé functions, respectively $[5,7]$. The algebraic form of all these functions is known but the parameters that they involve are not known, in any reasonable form, whenever $n \geq 4$. Hence, only ellipsoidal harmonics of degree less than or equal to 3 are known to possess simple analytic expressions [5, 7] and their particular form is given in Appendix A. The Lamé functions of the second kind $F_{n}^{m}$ are connected to the Lamé functions of the first kind $E_{n}^{m}$ through the formula

$$
\begin{aligned}
F_{n}^{m}(\rho) & =(2 n+1) E_{n}^{m}(\rho) \int_{\rho}^{+\infty} \frac{d x}{\left[E_{n}^{m}(x)\right]^{2} \sqrt{x^{2}-h_{2}^{2}} \sqrt{x^{2}-h_{3}^{2}}} \\
& =(2 n+1) E_{n}^{m}(\rho) I_{n}^{m}(\rho),
\end{aligned}
$$

for every $n=0,1,2, \ldots$ and $m=1,2, \ldots, 2 n+1$.

A key relation for our work is the expansion of the fundamental solution of Laplace's equation in terms of ellipsoidal harmonics which has been obtained in $[8,11,16]$ and assumes the form

$$
\frac{1}{\left|\mathbf{r}-\mathbf{r}^{\prime}\right|}=\sum_{n=0}^{\infty} \sum_{m=1}^{2 n+1} \frac{1}{(2 n+1) \gamma_{n}^{m}} \mathbb{E}_{n}^{m}\left(\rho^{\prime}, \mu^{\prime}, \nu^{\prime}\right) \mathbb{F}_{n}^{m}(\rho, \mu, \nu),
$$


where $\rho^{\prime}<\rho$ and

$$
\begin{aligned}
\gamma_{n}^{m} & =\frac{1}{4 \pi} \oint_{\rho=a_{1}} \frac{\left[E_{n}^{m}(\mu) E_{n}^{m}(\nu)\right]^{2}}{\sqrt{\left(a_{1}^{2}-\mu^{2}\right)\left(a_{1}^{2}-\nu^{2}\right)}} d s \\
& =\frac{2}{\pi} \int_{0}^{h_{3}} \int_{h_{3}}^{h_{2}} \frac{\left[E_{n}^{m}(\mu) E_{n}^{m}(\nu)\right]^{2}\left(\mu^{2}-\nu^{2}\right)}{\sqrt{\left(h_{2}^{2}-\mu^{2}\right)\left(h_{2}^{2}-\nu^{2}\right)\left(\mu^{2}-h_{3}^{2}\right)\left(h_{3}^{2}-\nu^{2}\right)}} d \mu d \nu
\end{aligned}
$$

denote the weighted norm integrals over the surface $S$ of the ellipsoid $\rho=a_{1}$.

The harmonic function that solves (15)-(16) is given for $\rho \leq a_{1}$ and, using (24), by

$$
u(\rho, \mu, \nu)=\sum_{n=0}^{\infty} \sum_{m=1}^{2 n+1} \frac{I_{n}^{m}\left(a_{1}\right)}{\gamma_{n}^{m}} \mathbb{E}_{n}^{m}\left(h_{2}, h_{3}, 0\right) \mathbb{E}_{n}^{m}(\rho, \mu, \nu),
$$

since the spherical limit $r \rightarrow 0+$ corresponds to the ellipsoidal limit $(\rho, \mu, \nu) \rightarrow\left(h_{2}, h_{3}, 0\right)$. Then by continuity, Eqs. (14) and (27) furnish the following formula:

$$
C^{\prime}=\sum_{n=0}^{\infty} \sum_{m=1}^{2 n+1} \frac{I_{n}^{m}\left(a_{1}\right)}{\gamma_{n}^{m}}\left[\mathbb{E}_{n}^{m}\left(h_{2}, h_{3}, 0\right)\right]^{2},
$$

which finally gives the capacity of the inverted triaxial ellipsoid.

In particular, for the ellipsoidal harmonics for which the exact form is known we have

$$
\begin{aligned}
\mathbb{E}_{0}^{1}\left(h_{2}, h_{3}, 0\right) & =1, \\
\mathbb{E}_{1}^{m}\left(h_{2}, h_{3}, 0\right) & =0, \quad m=1,2,3, \\
\mathbb{E}_{2}^{1}\left(h_{2}, h_{3}, 0\right) & =\left(\Lambda-a_{1}^{2}\right)\left(\Lambda-a_{2}^{2}\right)\left(\Lambda-a_{3}^{2}\right), \\
\mathbb{E}_{2}^{2}\left(h_{2}, h_{3}, 0\right) & =\left(\Lambda^{\prime}-a_{1}^{2}\right)\left(\Lambda^{\prime}-a_{2}^{2}\right)\left(\Lambda^{\prime}-a_{3}^{2}\right), \\
\mathbb{E}_{2}^{m}\left(h_{2}, h_{3}, 0\right) & =0, \quad m=3,4,5, \\
\mathbb{E}_{3}^{m}\left(h_{2}, h_{3}, 0\right) & =0, \quad m=1,2, \ldots, 7,
\end{aligned}
$$

where

$$
\left.\begin{array}{c}
\Lambda \\
\Lambda^{\prime}
\end{array}\right\}=\frac{a_{1}^{2}+a_{2}^{2}+a_{3}^{2}}{3} \pm \frac{1}{3} \sqrt{a_{1}^{4}+a_{2}^{4}+a_{3}^{4}-a_{1}^{2} a_{2}^{2}-a_{2}^{2} a_{3}^{2}-a_{3}^{2} a_{1}^{2}}
$$

are the two roots of

$$
\sum_{i=1}^{3} \frac{1}{\Lambda-a_{i}^{2}}=0
$$

Also, from the general form of the interior ellipsoidal harmonics $[5,7]$ we obtain that

$$
\mathbb{E}_{n}^{m}\left(h_{2}, h_{3}, 0\right)=0, \quad n=\text { odd }, m=1,2, \ldots, 2 n+1 .
$$

Formula (28) then assumes the form

$$
\begin{aligned}
C^{\prime}=\frac{I_{0}^{1}\left(a_{1}\right)}{\gamma_{0}^{1}} & +\frac{I_{2}^{1}\left(a_{1}\right)}{\gamma_{2}^{1}}\left[\left(\Lambda-a_{1}^{2}\right)\left(\Lambda-a_{2}^{2}\right)\left(\Lambda-a_{3}^{2}\right)\right]^{2} \\
& +\frac{I_{2}^{2}\left(a_{1}\right)}{\gamma_{2}^{2}}\left[\left(\Lambda^{\prime}-a_{1}^{2}\right)\left(\Lambda^{\prime}-a_{2}^{2}\right)\left(\Lambda^{\prime}-a_{3}^{2}\right)\right]^{2} \\
& +\sum_{n=2}^{\infty} \sum_{m=1}^{4 n+1} \frac{I_{2 n}^{m}\left(a_{1}\right)}{\gamma_{2 n}^{m}}\left[\mathbb{E}_{2 n}^{m}\left(h_{2}, h_{3}, 0\right)\right]^{2}
\end{aligned}
$$


The constant $\gamma_{0}^{1}([5]$, p. 469) assumes the value

$$
\gamma_{0}^{1}=1
$$

and $\gamma_{1}^{i}, i=1,2,3$ are given in [10] as

$$
\gamma_{1}^{i}=\frac{h_{1}^{2} h_{2}^{2} h_{3}^{2}}{3 h_{i}^{2}}, \quad i=1,2,3 .
$$

The expressions for the norms below are derived in Appendix B:

$$
\begin{aligned}
\gamma_{2}^{1} & =-\frac{2}{5}\left(\Lambda-\Lambda^{\prime}\right)\left(\Lambda-a_{1}^{2}\right)\left(\Lambda-a_{2}^{2}\right)\left(\Lambda-a_{3}^{2}\right), \\
\gamma_{2}^{2} & =\frac{2}{5}\left(\Lambda-\Lambda^{\prime}\right)\left(\Lambda^{\prime}-a_{1}^{2}\right)\left(\Lambda^{\prime}-a_{2}^{2}\right)\left(\Lambda^{\prime}-a_{3}^{2}\right), \\
\gamma_{2}^{6-i} & =\frac{h_{i}^{2}}{15} h_{1}^{2} h_{2}^{2} h_{3}^{2}, \quad i=1,2,3 .
\end{aligned}
$$

Substitution of (39)-(43) into (38) leads to the following (best possible) analytical expression for the capacity of the Kelvin-inverted triaxial ellipsoid:

$$
\begin{aligned}
C^{\prime}= & I_{0}^{1}\left(a_{1}\right) \\
& -\frac{5}{2\left(\Lambda-\Lambda^{\prime}\right)}\left[I_{2}^{1}\left(a_{1}\right)\left(\Lambda-a_{1}^{2}\right)\left(\Lambda-a_{2}^{2}\right)\left(\Lambda-a_{3}^{2}\right)-I_{2}^{2}\left(a_{1}\right)\left(\Lambda^{\prime}-a_{1}^{2}\right)\left(\Lambda^{\prime}-a_{2}^{2}\right)\left(\Lambda^{\prime}-a_{3}^{2}\right)\right] \\
& +\sum_{n=2}^{\infty} \sum_{m=1}^{4 n+1} \frac{I_{2 n}^{m}\left(a_{1}\right)}{\gamma_{2 n}^{m}}\left[\mathbb{E}_{2 n}^{m}\left(h_{2}, h_{3}, 0\right)\right]^{2}
\end{aligned}
$$

where

$$
I_{2 n}^{m}\left(a_{1}\right)=\frac{1}{2} \int_{0}^{+\infty} \frac{d x}{\left[E_{2 n}^{m}\left(\sqrt{x+a_{1}^{2}}\right)\right]^{2} \sqrt{x+a_{1}^{2}} \sqrt{x+a_{2}^{2}} \sqrt{x+a_{3}^{2}}},
$$

which is obtained via the substitution $x \rightarrow \sqrt{x+a_{1}^{2}}$ in (24).

In the special case where $a_{1}=a$ and $a_{2}=a_{3}=b$, the ellipsoid degenerates into a spheroid, which is characterized as prolate when $a>b$ and as oblate when $a<b$. In this case we obtain [1]:

$$
\begin{gathered}
I_{0}^{1}(a)= \begin{cases}\left(a^{2}-b^{2}\right)^{-1 / 2} \cosh ^{-1} \frac{a}{b}, & \text { for prolate, } \\
\left(b^{2}-a^{2}\right)^{-1 / 2} \cos ^{-1} \frac{a}{b}, & \text { for oblate, }\end{cases} \\
\Lambda-\Lambda^{\prime}=\frac{2}{3}\left(a^{2}-b^{2}\right), \\
I_{2}^{1}(a)=\frac{9}{4\left(a^{2}-b^{2}\right)^{2}}\left(I_{0}^{1}(a)-\frac{3 a}{2 a^{2}+b^{2}}\right), \\
I_{2}^{2}(a)=\frac{9}{4\left(a^{2}-b^{2}\right)^{2}}\left(\frac{1}{6} I_{0}^{1}(a)+\frac{a\left(2 a^{2}-5 b^{2}\right)}{18 b^{4}}\right) .
\end{gathered}
$$

Finally, for the case of the sphere, where $a_{1}=a_{2}=a_{3}=a$, we recover the well-known result $[3,6]$ that

$$
C^{\prime}=I_{0}^{1}(a)=\frac{1}{a}
$$


Appendix A: Ellipsoidal harmonics. The ellipsoidal coordinates $(\rho, \mu, \nu)$ are connected to the Cartesian coordinates $\left(x_{1}, x_{2}, x_{3}\right)$ via (17)-(21). Using relative terminology from the spherical coordinate system, $\rho$ specifies a particular ellipsoid and plays the role of the "radial" variable, while $\mu, \nu$ specify a particular 1-sheet and a particular 2-sheet hyperboloid respectively, and they play the role of the "angular" variables.

Separation of variables for Laplace's equation in the ellipsoidal system $[5,7]$ leads to the Lamé differential equation for each one of the variables $\rho, \mu$, and $\nu$. The Lamé functions, that solve these equations in the corresponding intervals of variation of $\rho, \mu$, and $\nu$, are exactly known for degrees less than or equal to three. For degrees greater than three their form is known, but the constants that enter into these expressions cannot be found exactly in a simple form, because they are solutions of irreducible polynomials of degree higher than three.

There is only one Lamé function of the first kind and degree zero and this is

$$
E_{0}^{1}(x)=1
$$

The corresponding Lamé function of the second kind is given by

$$
F_{0}^{1}(x)=\int_{x}^{+\infty} \frac{d u}{\sqrt{u^{2}-h_{2}^{2}} \sqrt{u^{2}-h_{3}^{2}}}=\frac{1}{2} \int_{x^{2}-a_{1}^{2}}^{+\infty} \frac{d y}{R(y)}
$$

where

$$
R(y)=\sqrt{y+a_{1}^{2}} \sqrt{y+a_{2}^{2}} \sqrt{y+a_{3}^{2}} .
$$

Then, the interior ellipsoidal harmonic of zeroth-degree is given by

$$
\mathbb{E}_{0}^{1}(\rho, \mu, \nu)=E_{0}^{1}(\rho) E_{0}^{1}(\mu) E_{0}^{1}(\nu)=1
$$

and the exterior is given by

$$
\mathbb{F}_{0}^{1}(\rho, \mu, \nu)=F_{0}^{1}(\rho) E_{0}^{1}(\mu) E_{0}^{1}(\nu)=I_{0}^{1}(\rho) \mathbb{E}_{0}^{1}(\rho, \mu, \nu)
$$

where

$$
I_{0}^{1}(\rho)=\frac{1}{2} \int_{\rho^{2}-a_{1}^{2}}^{+\infty} \frac{d y}{R(y)} .
$$

For degree one we obtain three kinds of Lamé functions, which are given by

$$
E_{1}^{m}(x)=\sqrt{\left|x^{2}-a_{1}^{2}+a_{m}^{2}\right|}, \quad m=1,2,3
$$

for the first kind, and by

$$
\begin{aligned}
F_{1}^{m}(x) & =3 E_{1}^{m}(x) \int_{x}^{+\infty} \frac{d u}{\left[E_{1}^{m}(u)\right]^{2} \sqrt{u^{2}-h_{2}^{2}} \sqrt{u^{2}-h_{3}^{2}}} \\
& =\frac{3}{2} E_{1}^{m}(x) \int_{x^{2}-a_{1}^{2}}^{+\infty} \frac{d u}{\left[E_{1}^{m}\left(\sqrt{y+a_{1}^{2}}\right)\right]^{2} R(y)} \\
& =3 I_{1}^{m}(x) E_{1}^{m}(x), \quad m=1,2,3
\end{aligned}
$$


for the second kind. Hence the interior ellipsoidal harmonics of the first degree are

$$
\begin{aligned}
& \mathbb{E}_{1}^{1}(\rho, \mu, \nu)=\rho \mu \nu, \\
& \mathbb{E}_{1}^{2}(\rho, \mu, \nu)=\sqrt{\rho^{2}-h_{3}^{2}} \sqrt{\mu^{2}-h_{3}^{2}} \sqrt{h_{3}^{2}-\nu^{2}}, \\
& \mathbb{E}_{1}^{3}(\rho, \mu, \nu)=\sqrt{\rho^{2}-h_{2}^{2}} \sqrt{h_{2}^{2}-\mu^{2}} \sqrt{h_{2}^{2}-\nu^{2}},
\end{aligned}
$$

and the corresponding exterior harmonics are

$$
\mathbb{F}_{1}^{m}(\rho, \mu, \nu)=F_{1}^{m}(\rho) E_{1}^{m}(\mu) E_{1}^{m}(\nu)=3 I_{1}^{m}(\rho) \mathbb{E}_{1}^{m}(\rho, \mu, \nu), \quad m=1,2,3
$$

where $I_{1}^{m}(\rho)$ is defined in (A.8).

Next we give the second-degree functions, which are of five different orders. The Lamé functions of the first kind are expressed in terms of the two constants $\Lambda, \Lambda^{\prime}$ given in (35) as solutions of (36). They are

$$
\begin{aligned}
& E_{2}^{1}(x)=x^{2}-a_{1}^{2}+\Lambda, \\
& E_{2}^{2}(x)=x^{2}-a_{1}^{2}+\Lambda^{\prime}, \\
& E_{2}^{3}(x)=x \sqrt{\left|x^{2}-a_{1}^{2}+a_{2}^{2}\right|} \\
& E_{2}^{4}(x)=x \sqrt{\left|x^{2}-a_{1}^{2}+a_{3}^{2}\right|}, \\
& E_{2}^{5}(x)=\sqrt{\left|x^{2}-a_{1}^{2}+a_{2}^{2}\right|} \sqrt{\left|x^{2}-a_{1}^{2}+a_{3}^{2}\right|} .
\end{aligned}
$$

The corresponding functions of the second order are

$$
\begin{aligned}
F_{2}^{m}(x) & =5 E_{2}^{m}(x) \int_{x}^{+\infty} \frac{d u}{\left[E_{2}^{m}(u)\right]^{2} \sqrt{u^{2}-h_{2}^{2}} \sqrt{u^{2}-h_{3}^{2}}} \\
& =\frac{5}{2} E_{2}^{m}(x) \int_{x^{2}-a_{1}^{2}}^{+\infty} \frac{d y}{\left[E_{2}^{m}\left(\sqrt{y+a_{1}^{2}}\right)\right]^{2} R(y)} \\
& =5 I_{2}^{m}(x) E_{2}^{m}(x), \quad m=1,2,3,4,5 .
\end{aligned}
$$

The interior ellipsoidal harmonics of the second degree are

$$
\begin{aligned}
& \mathbb{E}_{2}^{1}(\rho, \mu, \nu)=\left(\rho^{2}-a_{1}^{2}+\Lambda\right)\left(\mu^{2}-a_{1}^{2}+\Lambda\right)\left(\nu^{2}-a_{1}^{2}+\Lambda\right), \\
& \mathbb{E}_{2}^{2}(\rho, \mu, \nu)=\left(\rho^{2}-a_{1}^{2}+\Lambda^{\prime}\right)\left(\mu^{2}-a_{1}^{2}+\Lambda^{\prime}\right)\left(\nu^{2}-a_{1}^{2}+\Lambda^{\prime}\right), \\
& \mathbb{E}_{2}^{3}(\rho, \mu, \nu)=\rho \mu \nu \sqrt{\rho^{2}-h_{3}^{2}} \sqrt{\mu^{2}-h_{3}^{2}} \sqrt{h_{3}^{2}-\nu^{2}}, \\
& \mathbb{E}_{2}^{4}(\rho, \mu, \nu)=\rho \mu \nu \sqrt{\rho^{2}-h_{2}^{2}} \sqrt{h_{2}^{2}-\mu^{2}} \sqrt{h_{2}^{2}-\nu^{2}}, \\
& \mathbb{E}_{2}^{5}(\rho, \mu, \nu)=\sqrt{\rho^{2}-h_{2}^{2}} \sqrt{\rho^{2}-h_{3}^{2}} \sqrt{h_{2}^{2}-\mu^{2}} \sqrt{\mu^{2}-h_{3}^{2}} \sqrt{h_{2}^{2}-\nu^{2}} \sqrt{h_{3}^{2}-\nu^{2}},
\end{aligned}
$$

and the exterior ones are

$$
\mathbb{F}_{2}^{m}(\rho, \mu, \nu)=F_{2}^{m}(\rho) E_{2}^{m}(\mu) E_{2}^{m}(\nu)=5 I_{2}^{m}(\rho) \mathbb{E}_{2}^{m}(\rho, \mu, \nu), \quad m=1,2,3,4,5 .
$$

Finally, we give the highest-degree ellipsoidal harmonics that are known, in a simple form, that is, the seven harmonics of degree three. We introduce the following three 
pairs of parameters:

$$
\begin{aligned}
& \left.\begin{array}{c}
\Lambda_{n} \\
\Lambda_{n}^{\prime}
\end{array}\right\}=\frac{1}{5}\left(2 a_{1}^{2}+2 a_{2}^{2}+2 a_{3}^{2}-a_{n}^{2}\right) \\
& \pm \frac{1}{5} \sqrt{4 a_{1}^{4}+4 a_{2}^{4}+4 a_{3}^{4}-3 a_{n}^{4}-a_{1}^{2} a_{2}^{2}-a_{2}^{2} a_{3}^{2}-a_{3}^{2} a_{1}^{2}-6 a_{1}^{2} a_{2}^{2} a_{3}^{2} a_{n}^{-2}}, \quad n=1,2,3,
\end{aligned}
$$

which solve the three quadratic equations

$$
\sum_{i=1}^{3} \frac{1}{\Lambda_{n}-a_{i}^{2}}+\frac{2}{\Lambda_{n}-a_{n}^{2}}=0, \quad n=1,2,3 .
$$

In terms of the above parameters we obtain the seven Lamé functions of the third degree listed below:

$$
\begin{aligned}
& E_{3}^{1}(x)=x\left(x^{2}-a_{1}^{2}+\Lambda_{1}\right), \\
& E_{3}^{2}(x)=x\left(x^{2}-a_{1}^{2}+\Lambda_{1}^{\prime}\right), \\
& E_{3}^{3}(x)=x\left(x^{2}-a_{1}^{2}+\Lambda_{2}\right), \\
& E_{3}^{4}(x)=x\left(x^{2}-a_{1}^{2}+\Lambda_{2}^{\prime}\right), \\
& E_{3}^{5}(x)=x\left(x^{2}-a_{1}^{2}+\Lambda_{3}\right), \\
& E_{3}^{6}(x)=x\left(x^{2}-a_{1}^{2}+\Lambda_{3}^{\prime}\right), \\
& E_{3}^{7}(x)=x \sqrt{\left|x^{2}-a_{1}^{2}+a_{2}^{2}\right|} \sqrt{\left|x^{2}-a_{1}^{2}+a_{3}^{2}\right|} .
\end{aligned}
$$

Similarly the seven Lamé functions of the second kind are given by

$$
\begin{aligned}
F_{3}^{m}(x) & =7 E_{3}^{m}(x) \int_{x}^{+\infty} \frac{d u}{\left[E_{3}^{m}(u)\right]^{2} \sqrt{u^{2}-h_{2}^{2}} \sqrt{u^{2}-h_{3}^{2}}} \\
& =\frac{7}{2} E_{3}^{m}(x) \int_{x^{2}-a_{1}^{2}}^{+\infty} \frac{d y}{\left[E_{3}^{m}\left(\sqrt{y+a_{1}^{2}}\right)\right]^{2} R(y)} \\
& =7 I_{3}^{m}(x) E_{3}^{m}(x), \quad m=1,2,3,4,5,6,7 .
\end{aligned}
$$

Thus, one obtains the following expressions for the interior harmonics:

$$
\begin{aligned}
& \mathbb{E}_{3}^{1}(\rho, \mu, \nu)=\rho \mu \nu\left(\rho^{2}-a_{1}^{2}+\Lambda_{1}\right)\left(\mu^{2}-a_{1}^{2}+\Lambda_{1}\right)\left(\nu^{2}-a_{1}^{2}+\Lambda_{1}\right), \\
& \mathbb{E}_{3}^{2}(\rho, \mu, \nu)=\rho \mu \nu\left(\rho^{2}-a_{1}^{2}+\Lambda_{1}^{\prime}\right)\left(\mu^{2}-a_{1}^{2}+\Lambda_{1}^{\prime}\right)\left(\nu^{2}-a_{1}^{2}+\Lambda_{1}^{\prime}\right), \\
& \mathbb{E}_{3}^{3}(\rho, \mu, \nu)=\rho \mu \nu\left(\rho^{2}-a_{1}^{2}+\Lambda_{2}\right)\left(\mu^{2}-a_{1}^{2}+\Lambda_{2}\right)\left(\nu^{2}-a_{1}^{2}+\Lambda_{2}\right), \\
& \mathbb{E}_{3}^{4}(\rho, \mu, \nu)=\rho \mu \nu\left(\rho^{2}-a_{1}^{2}+\Lambda_{2}^{\prime}\right)\left(\mu^{2}-a_{1}^{2}+\Lambda_{2}^{\prime}\right)\left(\nu^{2}-a_{1}^{2}+\Lambda_{2}^{\prime}\right), \\
& \mathbb{E}_{3}^{5}(\rho, \mu, \nu)=\rho \mu \nu\left(\rho^{2}-a_{1}^{2}+\Lambda_{3}\right)\left(\mu^{2}-a_{1}^{2}+\Lambda_{3}\right)\left(\nu^{2}-a_{1}^{2}+\Lambda_{3}\right), \\
& \mathbb{E}_{3}^{6}(\rho, \mu, \nu)=\rho \mu \nu\left(\rho^{2}-a_{1}^{2}+\Lambda_{3}^{\prime}\right)\left(\mu^{2}-a_{1}^{2}+\Lambda_{3}^{\prime}\right)\left(\nu^{2}-a_{1}^{2}+\Lambda_{3}^{\prime}\right), \\
& \mathbb{E}_{3}^{7}(\rho, \mu, \nu)=\rho \mu \nu \sqrt{\rho^{2}-h_{2}^{2}} \sqrt{\rho^{2}-h_{3}^{2}} \sqrt{h_{2}^{2}-\mu^{2}} \sqrt{\mu^{2}-h_{3}^{2}} \sqrt{h_{2}^{2}-\nu^{2}} \sqrt{h_{3}^{2}-\nu^{2}},
\end{aligned}
$$

and for the exterior harmonics

$$
\mathbb{F}_{3}^{m}(\rho, \mu, \nu)=F_{3}^{m}(\rho) E_{3}^{m}(\mu) E_{3}^{m}(\nu)=7 I_{3}^{m}(\rho) \mathbb{E}_{3}^{m}(\rho, \mu, \nu), \quad m=1,2,3,4,5,6,7 .
$$


The elliptic integrals $I_{n}^{m}$ for $n=0,1,2,3$ and $m=1,2, \ldots,(2 n+1)$ can be expressed in terms of two independent integrals [1], for example, $I_{0}^{1}$ and $I_{1}^{1}$.

Appendix B: Evaluation of the norm integrals. The value of the surface norm integral of degree zero can be found in [7] (see also [5], p. 469) and is given by

$$
\gamma_{0}^{1}=1
$$

In order to evaluate the corresponding three norm integrals of degree one we proceed as follows. We know [1] that

$$
\begin{aligned}
{\left[E_{1}^{i}(\mu) E_{1}^{i}(\nu)\right]^{2}=} & \frac{h_{1}^{2} h_{2}^{2} h_{3}^{2}}{h_{i}^{2}} \frac{x_{i}^{2}}{a_{i}^{2}} \\
& =\frac{(-1)^{i}}{\Lambda-\Lambda^{\prime}}\left[\left(\Lambda^{\prime}-a_{i}^{2}\right) E_{2}^{1}(\mu) E_{2}^{1}(\nu)-\left(\Lambda-a_{i}^{2}\right) E_{2}^{2}(\mu) E_{2}^{2}(\nu)\right]+\frac{h_{1}^{2} h_{2}^{2} h_{3}^{2}}{3 h_{i}^{2}}, \\
i & =1,2,3 .
\end{aligned}
$$

Integrating (B.2) over the surface $\rho=a_{1}$ and using the orthogonality property of the surface ellipsoidal harmonics [5] with respect to the weighting function

$$
l(\mu, \nu)=\left(a_{1}^{2}-\mu^{2}\right)^{-1 / 2}\left(a_{1}^{2}-\nu^{2}\right)^{-1 / 2}
$$

as well as (A.1), we arrive at [10],

$$
\gamma_{1}^{i}=\frac{h_{1}^{2} h_{2}^{2} h_{3}^{2}}{3 h_{i}^{2}}, \quad i=1,2,3 .
$$

In a similar manner but with much more elaboration, we can evaluate the five norm integrals of degree two. Using again results from [1] and tedious calculations lead to

$$
\begin{aligned}
& {\left[E_{2}^{1}(\mu) E_{2}^{1}(\nu)\right]^{2}} \\
& =\frac{\left(\Lambda-a_{1}^{2}\right)^{2}\left(\Lambda-a_{2}^{2}\right)^{2}\left(\Lambda-a_{3}^{2}\right)^{2}}{\Lambda^{2}} \\
& \quad \cdot\left[\sum_{i=1}^{3} \frac{x_{i}^{4}}{\left(\Lambda-a_{i}^{2}\right)^{2}}+\frac{2 x_{1}^{2} x_{2}^{2} x_{3}^{2}}{\left(\Lambda-a_{1}^{2}\right)\left(\Lambda-a_{2}^{2}\right)\left(\Lambda-a_{3}^{2}\right)} \sum_{i=1}^{3} \frac{\Lambda-a_{i}^{2}}{x_{i}^{2}}+2 \sum_{i=1}^{3} \frac{x_{i}^{2}}{\Lambda-a_{i}^{2}}+1\right] \\
& {\left[E_{2}^{2}(\mu) E_{2}^{2}(\nu)\right]^{2}} \\
& =\frac{\left(\Lambda^{\prime}-a_{1}^{2}\right)^{2}\left(\Lambda^{\prime}-a_{2}^{2}\right)^{2}\left(\Lambda^{\prime}-a_{3}^{2}\right)^{2}}{\Lambda^{\prime 2}} \\
& \quad \cdot\left[\sum_{i=1}^{3} \frac{x_{i}^{4}}{\left(\Lambda^{\prime}-a_{i}^{2}\right)^{2}}+\frac{2 x_{1}^{2} x_{2}^{2} x_{3}^{2}}{\left(\Lambda^{\prime}-a_{1}^{2}\right)\left(\Lambda^{\prime}-a_{2}^{2}\right)\left(\Lambda^{\prime}-a_{3}^{2}\right)} \sum_{i=1}^{3} \frac{\Lambda^{\prime}-a_{i}^{2}}{x_{i}^{2}}+2 \sum_{i=1}^{3} \frac{x_{i}^{2}}{\Lambda^{\prime}-a_{i}^{2}}+1\right]
\end{aligned}
$$




$$
\begin{aligned}
& {\left[E_{2}^{3}(\mu) E_{2}^{3}(\nu)\right]^{2}=h_{1}^{2} h_{2}^{2} h_{3}^{4} \frac{x_{1}^{2} x_{2}^{2}}{a_{1}^{2} a_{2}^{2}},} \\
& {\left[E_{2}^{4}(\mu) E_{2}^{4}(\nu)\right]^{2}=h_{1}^{2} h_{2}^{4} h_{3}^{2} \frac{x_{1}^{2} x_{3}^{2}}{a_{1}^{2} a_{3}^{2}},} \\
& {\left[E_{2}^{5}(\mu) E_{2}^{5}(\nu)\right]^{2}=h_{1}^{4} h_{2}^{2} h_{3}^{2} \frac{x_{2}^{2} x_{3}^{2}}{a_{2}^{2} a_{3}^{2}},}
\end{aligned}
$$

From [1] we also obtain the following third-degree relations:

$$
\begin{aligned}
&\left.x_{i} x_{j}^{2}\right|_{\rho=a_{1}}= \frac{h_{i} a_{i} a_{j}^{2}}{h_{1} h_{2} h_{3}}\left[\frac{1+2 \delta_{i j}}{5} E_{1}^{i}(\mu) E_{1}^{i}(\nu)\right. \\
&\left.+\frac{(-1)^{j} h_{j}^{2}}{h_{1}^{2} h_{2}^{2} h_{3}^{2}} \frac{1}{\Lambda_{i}-\Lambda_{i}^{\prime}}\left(\left(\Lambda_{i}^{\prime}-a_{j}^{2}\right) E_{3}^{2 i-1}(\mu) E_{3}^{2 i-1}(\nu)-\left(\Lambda_{i}-a_{j}^{2}\right) E_{3}^{2 i}(\mu) E_{3}^{2 i}(\nu)\right)\right], \\
& i, j=1,2,3,
\end{aligned}
$$

which, when combined with the first-degree relations

$$
\left.x_{i}\right|_{\rho=a_{1}}=\frac{h_{i} a_{i}}{h_{1} h_{2} h_{3}} E_{1}^{i}(\mu) E_{1}^{i}(\nu), \quad i=1,2,3,
$$

furnish, by virtue of orthogonality, the values for the following surface integrals:

$$
\begin{gathered}
\int_{\rho=a_{1}} x_{i}^{2} l(\mu, \nu) d s=\frac{4 \pi}{3} a_{i}^{2}, \quad i=1,2,3 \\
\int_{\rho=a_{1}} x_{i}^{2} x_{j}^{2} l(\mu, \nu) d s=\frac{4 \pi}{15} a_{i}^{2} a_{j}^{2}, \quad i, j=1,2,3, \quad i \neq j \\
\int_{\rho=a_{1}} x_{i}^{4} l(\mu, \nu) d s=\frac{4 \pi}{5} a_{i}^{4}, \quad i=1,2,3 .
\end{gathered}
$$

Then (B.5), in view of (B.12)-(B.14), provides

$$
\begin{aligned}
\gamma_{2}^{1} & =\frac{\left(\Lambda-a_{1}^{2}\right)^{2}\left(\Lambda-a_{2}^{2}\right)^{2}\left(\Lambda-a_{3}^{2}\right)^{2}}{\Lambda^{2}} \\
. & {\left[\frac{1}{5} \sum_{i=1}^{3} \frac{a_{i}^{4}}{\left(\Lambda-a_{i}^{2}\right)^{2}}+\frac{2}{15} \frac{a_{1}^{2} a_{2}^{2} a_{3}^{2}}{\left(\Lambda-a_{1}^{2}\right)\left(\Lambda-a_{2}^{2}\right)\left(\Lambda-a_{3}^{2}\right)} \sum_{i=1}^{3} \frac{\Lambda-a_{i}^{2}}{a_{i}^{2}}+\frac{2}{3} \sum_{i=1}^{3} \frac{a_{i}^{2}}{\Lambda-a_{i}^{2}}+1\right] . }
\end{aligned}
$$

Straightforward calculations also show that

$$
\begin{gathered}
\sum_{i=1}^{3} \frac{a_{i}^{2}}{\Lambda-a_{i}^{2}}=-3 \\
\frac{2 a_{1}^{2} a_{2}^{2} a_{3}^{2}}{\left(\Lambda-a_{1}^{2}\right)\left(\Lambda-a_{2}^{2}\right)\left(\Lambda-a_{3}^{2}\right)} \sum_{i=1}^{3} \frac{\Lambda-a_{i}^{2}}{a_{i}^{2}}=9-\sum_{i=1}^{3} \frac{a_{i}^{4}}{\left(\Lambda-a_{i}^{2}\right)^{2}},
\end{gathered}
$$


and

$$
\sum_{i=1}^{3} \frac{a_{i}^{4}}{\left(\Lambda-a_{i}^{2}\right)^{2}}=3-\frac{3 \Lambda^{2}\left(\Lambda-\Lambda^{\prime}\right)}{\left(\Lambda-a_{1}^{2}\right)\left(\Lambda-a_{2}^{2}\right)\left(\Lambda-a_{3}^{2}\right)}
$$

Substituting (B.16)-(B.18) into (B.15) finally renders

$$
\gamma_{2}^{1}=-\frac{2}{5}\left(\Lambda-\Lambda^{\prime}\right)\left(\Lambda-a_{1}^{2}\right)\left(\Lambda-a_{2}^{2}\right)\left(\Lambda-a_{3}^{2}\right)
$$

A series of similar calculations leads to

$$
\begin{aligned}
\gamma_{2}^{2} & =\frac{2}{5}\left(\Lambda-\Lambda^{\prime}\right)\left(\Lambda^{\prime}-a_{1}^{2}\right)\left(\Lambda^{\prime}-a_{2}^{2}\right)\left(\Lambda^{\prime}-a_{3}^{2}\right), \\
\gamma_{2}^{3} & =\frac{1}{15} h_{1}^{2} h_{2}^{2} h_{3}^{4}, \\
\gamma_{2}^{4} & =\frac{1}{15} h_{1}^{2} h_{2}^{4} h_{3}^{2} \\
\gamma_{2}^{5} & =\frac{1}{15} h_{1}^{4} h_{2}^{2} h_{3}^{2} .
\end{aligned}
$$

Hence, all the surface norm integrals of degree less than or equal to two have been evaluated.

Acknowledgment. This work was initiated during a short visit of the first author to the University of Tel Aviv, under the Greece-Israel bilateral scientific exchange program. The first author acknowledges fruitful discussion with Dr. Antonios Charalambopoulos, mainly with the material appearing in Appendix B.

\section{REFERENCES}

[1] G. Dassios, Scattering of acoustic waves by a coated pressure-Release ellipsoid, J.A.S.A. 70, 176$185(1981)$

[2] G. Dassios, On the harmonic radius and the capacity of an inverse ellipsoid, J. Math. Phys. 27, 835-836 (1988)

[3] G. Dassios and R. E. Kleinman, On Kelvin inversion and low-frequency scattering, SIAM Review 31, 565-585 (1989)

[4] G. Dassios and R. E. Kleinman, On the capacity and Rayleigh scattering for a class of nonconvex bodies, Quart. J. Mech. Appl. Math. 42, 467-475 (1989)

[5] E. W. Hobson, The Theory of Spherical and Ellipsoidal Harmonics, Chelsea, New York, 1955

[6] R. E. Kleinman and T. B. A. Senior, Rayleigh Scattering, in Low and High Frequency Asymptotics, V. K. Varadan and V. V. Varadan, Eds., North-Holland, Amsterdam, 1986

[7] W. D. MacMilan, The Theory of the Potential, Dover, New York, 1958

[8] T. Miloh, Forces and moments on a tri-axial ellipsoid in potential flow, Israel J. Techn. 11, 63-74 (1973)

[9] T. Miloh, The ultimate image singularities for external ellipsoidal harmonics, SIAM Appl. Math. 26, 334-344 (1973)

[10] T. Miloh, Maneuvering hydrodynamics of ellipsoidal forms, J. Ship Res. 23 66-75 (1979)

[11] B. D. Sleeman, The low-frequency scalar Dirichlet scattering by a general ellipsoid, IMA J. of Appl. Math. 3, 291-312 (1967)

[12] A. F. Stevenson, Solution of electromagnetic scattering problems as power series in the ratio (dimension of scatterer/wave length), J. Appl. Phys. 24, 1134-1142 (1953) 
[13] J. W. Strutt, Lord Rayleigh, On the incidence of aerial and electric waves upon small obstacles in the form of ellipsoids or elliptic cylinders and on the passage of electric waves through a circular aperture in a conducting screen, Philos. Mag. 44, 28-52 (1897)

[14] W. Thomson, Lord Kelvin, Papers on Electrostatics and Magnetism, MacMillan, London, 1882. First Published in J. Math. Pure Appl. 10, p. 364 (1845) and 12, p. 256 (1847)

[15] V. Twersky, Rayleigh Scattering, Appl. Opt. 3, 1150-1162 (1964)

[16] W. E. Williams, Some results for low-frequency Dirichlet scattering by arbitrary obstacles and their application to the particular case of the ellipsoid, IMA J. of Appl. Math. 7, 111-118 (1971) 